This is an electronic reprint of the original article. This reprint may differ from the original in pagination and typographic detail.

\author{
Author(s): Lauter, Hans; Apaja, Vesa; Kalinin, I.; Kats, E.; Koza, M.; Krotscheck, E.; Lauter, V.V.; \\ Puchkov, A.V.
}

Title: $\quad$ Observation of a superfluid component within solid helium

Year: $\quad 2011$

Version:

Please cite the original version:

Lauter, H., Apaja, V., Kalinin, I., Kats, E., Koza, M., Krotscheck, E., Lauter, V.V., \& Puchkov, A.V. (2011). Observation of a superfluid component within solid helium. Physical Review Letters, 107(26), 4. https://doi.org/10.1103/PhysRevLett.107.265301

All material supplied via JYX is protected by copyright and other intellectual property rights, and duplication or sale of all or part of any of the repository collections is not permitted, except that material may be duplicated by you for your research use or educational purposes in electronic or print form. You must obtain permission for any other use. Electronic or print copies may not be offered, whether for sale or otherwise to anyone who is not an authorised user. 


\title{
Observation of a Superfluid Component within Solid Helium
}

\author{
H. Lauter, ${ }^{1}$ V. Apaja, ${ }^{2}$ I. Kalinin, ${ }^{3}$ E. Kats, ${ }^{4,5}$ M. Koza, ${ }^{4}$ E. Krotscheck, ${ }^{6,7}$ V. V. Lauter, ${ }^{1}$ and A. V. Puchkov ${ }^{3}$ \\ ${ }^{1}$ Spallation Neutron Source, Oak Ridge National Laboratory, Oak Ridge, Tennessee 37831, USA \\ ${ }^{2}$ Nanoscience Center, University of Jyväskylä, Finland \\ ${ }^{3}$ Institute for Physics and Power Engineering, 249020 Bondarenko sq. 1, Obninsk, Russia \\ ${ }^{4}$ Institut Laue-Langevin, 6, rue Jules Horowitz, 38042 Grenoble, France \\ ${ }^{5}$ L. D. Landau Institute for Theoretical Physics, Russian Academy of Sciences, Moscow, Russia \\ ${ }^{6}$ Institut für Theoretische Physik, Johannes Kepler Universität, 4040 Linz, Austria \\ ${ }^{7}$ Department of Physics, University at Buffalo SUNY, Buffalo New York, 14260, USA
}

(Received 24 September 2010; revised manuscript received 11 April 2011; published 22 December 2011)

\begin{abstract}
We demonstrate by neutron scattering that a localized superfluid component exists at high pressures within solid helium in aerogel. Its existence is deduced from the observation of two sharp phonon-roton spectra which are clearly distinguishable from modes in bulk superfluid helium. These roton excitations exhibit different roton gap parameters than the roton observed in the bulk fluid at freezing pressure. One of the roton modes disappears after annealing the samples. Comparison with theoretical calculations suggests that the model that reproduces the observed data best is that of superfluid double layers within the solid and at the helium-substrate interface.
\end{abstract}

DOI: 10.1103/PhysRevLett.107.265301

PACS numbers: 67.25.dr, 67.80.bd, 67.90.+z

Neutron scattering experiments play an important role in the investigation of quantum fluids. They provide, among others, information on the presence and nature of a superfluid state on a microscopic scale. Of particular interest are the roton excitations in superfluid helium with their characteristic minimum in the dispersion relation. The "roton gap" has been a key quantity since the first explanation of superfluidity [1]. Roton data were used to characterize excitations in helium in nanoporous gelsil $[2,3]$ and in a single helium crystal experiment [4].

We report in this Letter the observation of two rotonlike excitations in solid ${ }^{4} \mathrm{He}$ in aerogel at pressures far above the melting pressure. The observation of these modes demonstrates the existence of a localized superfluid component in equilibrium with the solid. We also provide a microscopic interpretation of these modes.

The initial configuration of the solid helium was intended to be a perturbed polycrystal. To achieve this, we have condensed the helium within the confinement of a aerogel matrix $[5,6]$, and cooled the sample at constant density $[7,8]$ with the available cooling rate of the cryostat [9]. The purpose of the aerogel matrix is to generate a large surface area and numerous dislocations, who act as nucleation sites for helium crystallites. The first set of neutron scattering experiments was conducted for the so-prepared solid helium at the lowest temperatures.

We have carried out neutron scattering measurements on this sample at pressures of 37.0, 44.8, and 53.8 bar and a temperature of $50 \mathrm{mK}$ [10]. The resulting intensity maps are shown in Fig. 1. The pressures in the sample cell were derived by interpolating the measured hcp lattice parameters $[11,12]$. We have obtained, as wanted, a powder spectrum, deduced from our elastic scattering events in Fig. 1.
The confinement in aerogel suggests the unusual coexistence of small bcc and hep crystallites that lets us expect further changes in the quantum behavior of this system [13].

The eye-catching feature is the inelastic scattering intensity along two dispersion lines that both resemble a phonon-roton spectrum in bulk superfluid helium. We will refer to these two modes with different roton gap energy $E_{\Delta}$ and roton wave number $Q_{\Delta}$ as "upper" and "lower" rotons corresponding to their energy $E_{\Delta}$.

The similarity of the upper spectrum to a bulk phononroton spectrum is striking, but the modes are clearly different from the bulk ones:

(i) Both modes are nearly independent on pressure.

(ii) Both modes show roton gaps with parameters distinctly different from those of bulk helium at the melting pressure of 25 bar.

Thus, these excitations can not be due to rotons propagating in bulk ${ }^{4} \mathrm{He}$. Moreover, the observed phonon-roton spectra show the instrumental resolution, i.e., a sharp intrinsic helium signal, which is characteristic for a superfluid. A normal fluid would show a substantial energy broadening due to quasielastic scattering. Hence, the measured excitation branches must originate from confined superfluid helium components within the sample.

Figure 1 shows the pressure dependence of the two modes as well as that of the structure of the solid. From the appearance of Bragg peaks we see that coexisting bcc and hcp phases emerge with increasing pressure. The hcp phase gains considerable intensity at 53.8 bar, whereas the intensity of the bcc phase is reduced [14]. The integrated intensity of the Bragg peaks increases with pressure. Along with that, the intensity of the upper roton decreases, 


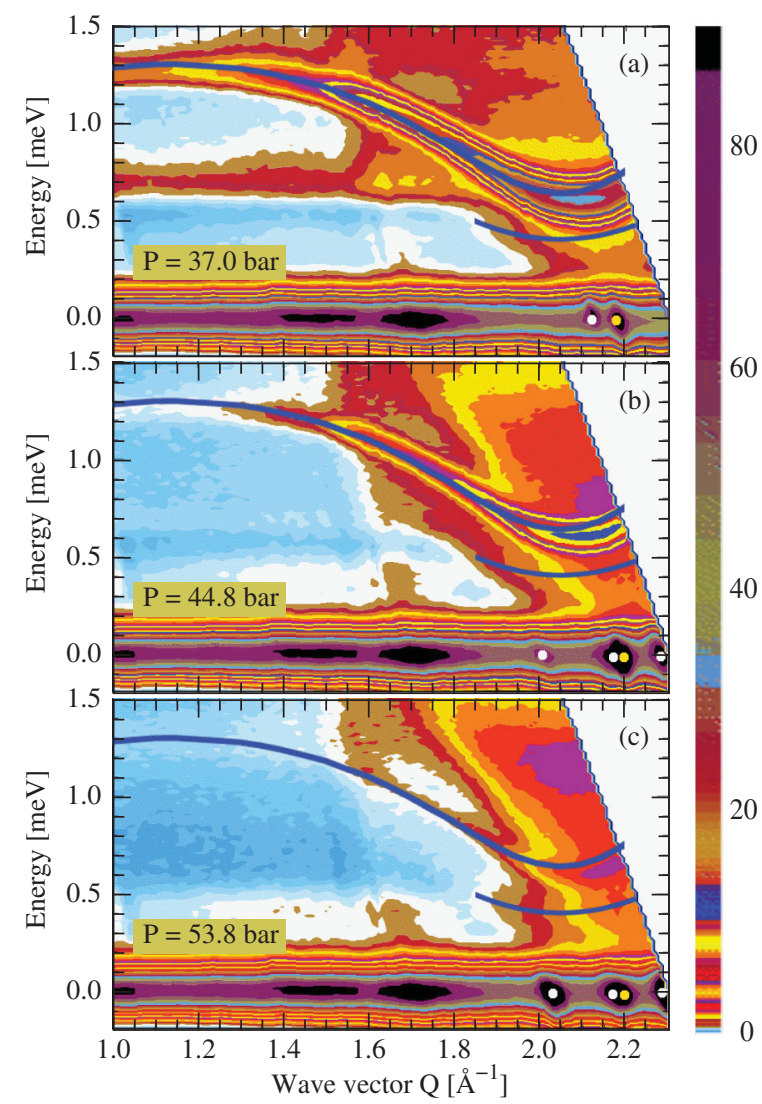

FIG. 1 (color). Intensity maps of inelastic and elastic neutron scattering data taken on the time-of-flight spectrometer IN6 at ILL [23] at a temperature of $50 \mathrm{mK}$ at three pressures. The colorscale has been normalized to the elastic scattering intensity of the aerogel. The diffraction and small angle scattering from the aerogel appear in pink-black along the zero energy transfer line, $E=0 \mathrm{meV}$, as the Bragg-peaks of solid helium polycrystals for $Q>1.9 \AA^{-1}$. No scattering from amorphous solid helium was detected. The Bragg peaks are attributed tentatively to hcp and bcc bulk structures, marked by white and yellow circles, respectively. An average crystallite diameter of $50 \mathrm{~nm}$ is obtained for all structures from the diffraction peaks [14]. For comparison we also show the dispersion relation of bulk liquid helium at $T=0.5 \mathrm{~K}$ and $20 \mathrm{bar}$ (upper blue lines) [20]. The lower blue lines represent rotonlike intensities already known from helium films on aerogel [6] that can be identified as a "layer roton." Phonons emerging from the bulk helium Bragg peaks within the measured $Q$ range were not detected [9]. The signal with highest intensity around $E=1.2 \mathrm{meV}, Q=2.1 \AA^{-1}$ is proportional to the amount of solid helium [14].

whereas the intensity of the lower roton does not change; see Fig. 2. The ratio of the amount of confined liquid that contributes to the strength of the upper roton, to the total amount of solid helium as deduced from the scattered neutron intensities, is inversely proportional to the pressure and extrapolates to zero at about 56 bar. The upper roton must therefore be due to an excitation in a superfluid component that is reduced with increasing pressure. The intensity of the lower roton is, on the other hand,

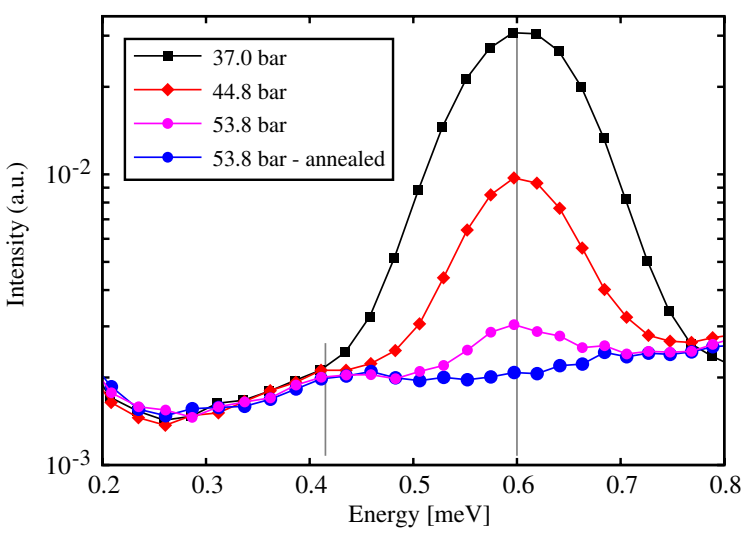

FIG. 2 (color online). Intensity cuts along $Q=2.09 \AA^{-1}$, taken from Figs. 1(a)-1(c). Also shown is the effect of annealing from Fig. 3(a). The gray lines mark the intensity maxima.

independent on pressure in the investigated pressure range, it must therefore be related to the aerogel substrate-solidhelium interface, the area of which is independent of pressure. Figure 2 highlights the different pressure dependence of these two modes.

A second important result beyond the discovery of the two roton spectra itself is that one roton spectrum, i.e., a superfluid component in solid helium, can be made to disappear by annealing: The sample at the highest pressure [Fig. 1(c)] was heated from $T=50 \mathrm{mK}$ to $T>1.3 \mathrm{~K}$ and then recooled to $T=600 \mathrm{mK}$. The striking effect shown in Fig. 3(a) is that the inelastic scattering strength along the upper roton dispersion curve vanished completely. This intensity, i.e., the corresponding superfluid component, returns after heating the sample to $3.5 \mathrm{~K}$ followed by recooling and, thus, reforming the solid phase.

The effect of annealing is highlighted in Fig. 3(b). There we show the difference between the scattered intensity of Fig. 1(c) and the one of Fig. 3(a). The dispersion curve obtained that way describes exclusively the excitations in the phase that has disappeared at annealing. The dispersion curve is the same as the one seen in Figs. 1(a)-1(c). The clear appearance of the intensity of only one mode shows independent scattering from only that phase.

To summarize the experiments, we have discovered the existence of two independent superfluid phases. One lives within the crystallites of solid helium, it disappears by annealing. The other one lives at the interface between solid helium and aerogel. The results were confirmed in a second experiment with the same sample [14].

The localized superfluid phases found in our experiments demonstrate a necessary but not a sufficient condition for superflow. Superflow manifests itself in the effect of nonclassical rotational inertia (NCRI), which is probed in torsional oscillator (TO) experiments, see, e.g., Kim and Chan [15]. In particular, superflow can be suppressed in TO studies at a $T_{c}$ around $100 \mathrm{mK}$. We observed no noticeable effect in the neutron data, when crossing this $T_{c}$ and neither 


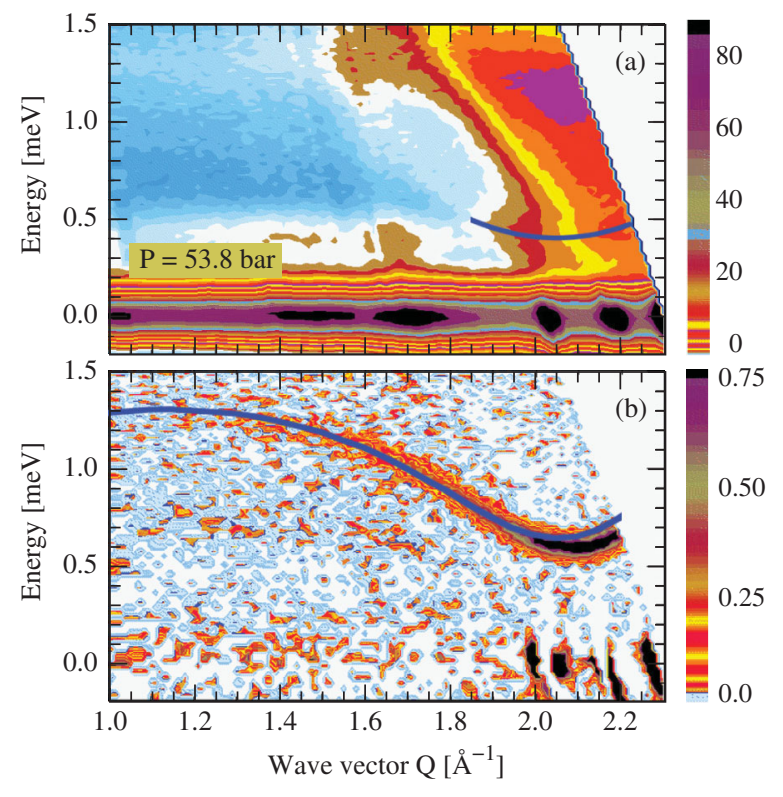

FIG. 3 (color). The effect of annealing at $\sim 1.3 \mathrm{~K}$ and 53.8 bar. The spectrum in pane (a) has been taken at $600 \mathrm{mK}$ after heating the sample from $50 \mathrm{mK}$ to $1.3 \mathrm{~K}$, followed by recooling back to $600 \mathrm{mK}$. The upper dispersion line, which we identify with excitations in superfluid double layers, has vanished. We identify the remaining lower roton with an excitation that is propagating in superfluid double layers at the aerogel-solid-helium interface. Pane (b) is generated by subtracting the intensities of Fig. 3(a) from those of Fig. 1(c), selecting the upper roton excitation which has vanished by annealing. The dark streaks in Fig. 3(b) around zero energy come from a slight shift of the Bragg peaks during annealing [14].

up to $1 \mathrm{~K}$ close to the annealing temperature. This is clear because our experiment displays only the signature of a superfluid component, the local superfluid order, but not the signature of superflow, i.e., the long-range coherent order over the volume of the sample cell, which is probed in TO experiments.

The detailed mechanism of supersolidity is still under discussion, the unknown structural composition of solid helium in different TO experiments seems to be the reason for partially contradictory results $[7,8]$.

Our findings are consistent with those of Rittner and Reppy $[16,17]$ who also find that the NCRI vanishes after annealing. Note again, that neutron scattering experiments can detect localized superfluid component but do not probe directly the superflow of NCRI. Our aim was to extend the data taking beyond $1 \mathrm{~K}$ to meet the temperature range where the annealing procedures of solid helium in the TO experiments by Rittner and Reppy [16] were performed, which initiated the discussion about imperfections and impurities in the solid helium [17].

We now turn to an interpretation of our data. Confined liquids are known to form layers [18,19], and comparison to microscopic calculations, to be presented below, points more precisely to superfluid double layers.
We first note that the intensity of the lower roton in Fig. 3(a) is not affected by annealing, also demonstrated in Fig. 2. This evidences the fact that this mode propagates at the interface between aerogel and solid helium, where the aerogel substrate stabilizes its configuration against higher pressures and higher temperatures. Consequently, we can also conclude that the upper phonon-roton excitations propagate in a fluid component inside the solid helium itself.

We compare in Fig. 4 the roton dispersion relations $E(Q)$ of the confined superfluid phases with $E_{\Delta}(p)$ and $Q_{\Delta}(p)$ of bulk helium [20] as a function of pressure to characterize its nature. Evidently, the two roton dispersion relations $E(Q)$ depend only weakly on pressure. Hence, any extrapolation of bulk roton parameters $E_{\Delta}, Q_{\Delta}$ to the pressures where the roton gaps of the new superfluid phases have been measured, leads to a significant discrepancy. Thus this discrepancy between the $E_{\Delta}$ and $Q_{\Delta}$ of the confined phases and the extrapolated bulk parameters suggests the existence of new confined superfluid phases.

The pressure insensitivity of the lower roton is caused by the interaction with the strongly attractive substrate which is attenuated by a solid layer of helium similar to helium films on graphite [18]. However, the upper roton should feel the changing pressure of the surrounding solid helium, since the hcp structure exhibits clear pressure induced changes. Since this is not the case, the important consequence is that also the interactions, which stabilize the

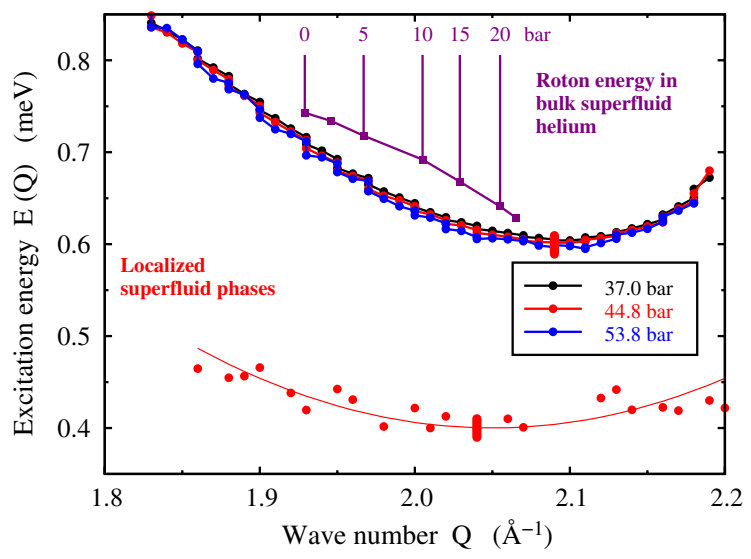

FIG. 4 (color online). The figure shows the measured roton dispersion relation $E(Q)$ in the confined phases for the three pressures given in Fig. 1. The curves represent results of fits to the $Q$-sliced intensity maps of Fig. 1(a)-1(c). Examples of the slices I (energy) are shown in Fig. 2. The energy of the roton minimum $E_{\Delta}$ and wave number $Q_{\Delta}$ in the bulk liquid is also shown (magenta line) as a function of pressure. It follows a line that is clearly distinct from the roton minimum of the two localized phases, which are nearly independent of pressure. Only a slight shift of its $E_{\Delta}$ of only $4.7 \mu \mathrm{eV} / 10$ bar is noticed as opposed to $\approx 49 \mu \mathrm{eV} / 10$ bar in the bulk phase. Also the $E_{\Delta}$, of the lower roton, whose energy is much lower than the roton gap in the bulk liquid, is, within statistics, independent of pressure. 


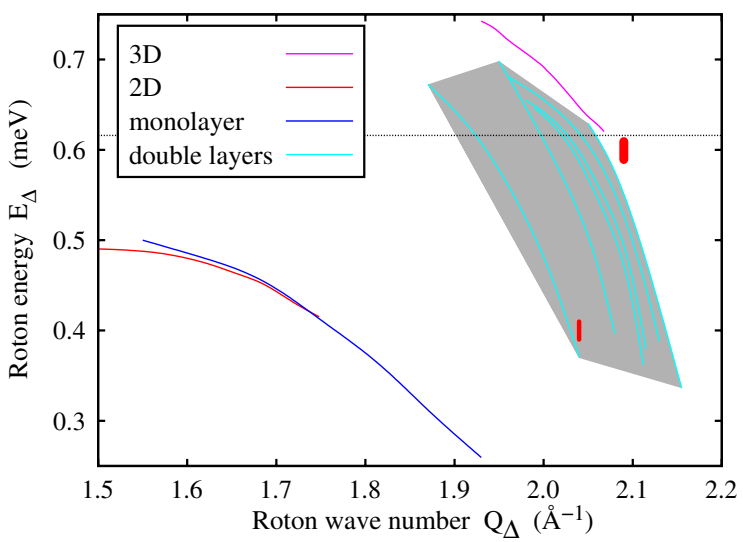

FIG. 5 (color online). Dependence of the roton parameters $E_{\Delta}$ and $Q_{\Delta}$ on pressure in various geometries. The measured values are represented by the two heavy red bars; see Fig. 4 . The light blue lines in the grey shaded area show a family of double-layer configurations where the solid substrate has been modeled by a 3-9 potential of well depth between 4 and $30 \mathrm{~K}$. The red curve shows theoretical results for a 2D model, which agree quite well with results for an atomic monolayer (blue curve). We also show $E_{\Delta}$ and $Q_{\Delta}$ of bulk liquid helium [20] as a function of pressure up to the melting line (magenta line). The horizontal dotted line shows the limiting energy of the bulk roton at melting pressure at $0.62 \mathrm{meV}$.

liquid double layers, are not changing with pressure due to the confinement conditions. Hence it is the amount of liquid carrying the upper roton, which is decreasing along with the intensity decrease with increasing pressure (Fig. 1).

Quantitative microscopic many-body calculations [19] have been performed to search for configurations of liquid helium whose excitations meet the experimentally determined spectra. We have studied, similar to Ref. [19], the nature and the excitations of configurations of liquid ${ }^{4} \mathrm{He}$ between two rigid walls interacting with the liquid through the usual 3-9 potentials. Given the uncertainty of this procedure, we have studied a range of liquid-solid interactions characterized by well depths between $4 \mathrm{~K}$ and $30 \mathrm{~K}$. We show in Fig. 5 the calculated dependence of the roton energy $E_{\Delta}$ on the roton wave number $Q_{\Delta}$ as a function of density for a number of such model systems: Rigorously 2D helium, a quasi 2D-monolayer, several double-layer configurations defined by a family of external fields, and bulk helium. The excitations in the quasi-2D monolayer are expectedly independent of the well depth of the potential.

According to these calculations, the energy of $E_{\Delta}=$ $0.40 \mathrm{meV}$ of the interface mode between the aerogel and the solid helium can be explained by either a liquid monolayer or a high density double layer. The corresponding roton wave number $Q_{\Delta} \approx 2.04 \AA^{-1}$ can, however, not be associated with an excitation propagating in a liquid monolayer; hence we conclude that there must be at least two superfluid layers between the aerogel and solid helium.
The $E_{\Delta}=0.60 \mathrm{meV}$ of the upper roton can also only be explained by a liquid double layer.

Our results are consistent with the simulations of Khairallah and Ceperley [21] who show the existence of superfluid layers at the interface between Vycor and solid ${ }^{4} \mathrm{He}$. PIMC simulations [22] suggest triple layers as grain boundary structure. However, in such a geometry one would also have a multitude of rotonlike excitations with higher $E_{\Delta i} ; Q_{\Delta i}$ [9]. Having only one dispersion curve in the experimental data, we conclude that the upper roton propagates in a superfluid double layer sandwiched by solid helium. As seen in Fig. 5, all potential models lead to the same conclusion.

Thus, the nature of the detected superfluid state has been clarified by microscopic many-body calculations, reproducing the roton gaps of the two excitation branches. The calculations show that the detected superfluid components correspond to quasi-2D liquid double layers, one sandwiched between areas of stressed solid helium and the other one between the aerogel substrate and solid helium. Both excitations are analogous to the layer-rotons in superfluid helium films on graphite [18] or aerogel [6].

This work was supported, in part, by RFBR grant 09-0297501 Amadée Project No. FR 06/2008, Finnish Academy project 7115066 and the FWF Project P21264. Discussions with C.E. Campbell, F. M. Gasparini, H. Godfrin, and K. B. Whaley are also acknowledged.

[1] L. Landau, J. Phys. USSR 11, 91 (1947).

[2] J. V. Pearce, J. Bossy, H. Schober, H. R. Glyde, D. R. Daughton, and N. Mulders, Phys. Rev. Lett. 93, 145303 (2004).

[3] J. Bossy, J. V. Pearce, H. Schober, and H. R. Glyde, Phys. Rev. Lett. 101, 025301 (2008).

[4] E. Blackburn, J. Goodkind, S. K. Sinha, C. Broholm, J. Copley, and R. Erwin, PRAMANA- J. Phys. 71, 673 (2009).

[5] N. Mulders, J.T. West, M.H.W. Chan, C.N. Kodituwakku, C. A. Burns, and L. B. Lurio, Phys. Rev. Lett. 101, 165303 (2008).

[6] H. J. Lauter, I. V. Bogoyavlenskii, A. V. Puchkov, H. Godfrin, A. Skomorokhov, J. Klier, and P. Leiderer, Appl. Phys. A 74, s1547 (2002).

[7] A. C. Clark, J. T. West, and M.H. W. Chan, Phys. Rev. Lett. 99, 135302 (2007).

[8] S. Sasaki, F. Caupin, and S. Balibar, J. Low Temp. Phys. 153, 43 (2008).

[9] See Supplemental Material at http://link.aps.org/supplemental/10.1103/PhysRevLett.107.265301 for details on the sample preparation, cooling, and theoretical analysis.

[10] I. V. Kalinin, E. Kats, M. Koza, V. V. Lauter, H. Lauter, and A. V. Puchkov, J. Exp. Theor. Phys. 111, 215 (2010).

[11] H. Wiechert, H. J. Lauter, and B. Stuehn, J. Low Temp. Phys. 48, 209 (1982).

[12] D. G. Henshaw, Phys. Rev. 109, 328 (1958). 
[13] D. W. Brown, P.E. Sokol, and S. N. Ehrlich, Phys. Rev. Lett. 81, 1019 (1998).

[14] V. Apaja, E. Krotscheck, and H.-J. Lauter (to be published).

[15] E. Kim and M.H.W. Chan, Nature (London) 427, 225 (2004).

[16] A. C. Rittner and J. D. Reppy, Phys. Rev. Lett. 97, 165301 (2006).

[17] E. S. Reich, Nature (London) 468, 748 (2010).

[18] B. E. Clements, H. Forbert, E. Krotscheck, H. J. Lauter, M. Saarela, and C. J. Tymczak, Phys. Rev. B 50, 6958 (1994).
[19] V. Apaja and E. Krotscheck, Phys. Rev. B 64, 134503 (2001).

[20] M. R. Gibbs, K.H. Andersen, W. G. Stirling, and H. Schober, J. Phys. Condens. Matter 11, 603 (1999).

[21] S. A. Khairallah and D. M. Ceperley, Phys. Rev. Lett. 95, 185301 (2005).

[22] L. Pollet, M. Boninsegni, A. B. Kuklov, N. V. Prokofev, B. V. Svistunov, and M. Troyer, Phys. Rev. Lett. 98, 135301 (2007).

[23] www.ill.eu. 